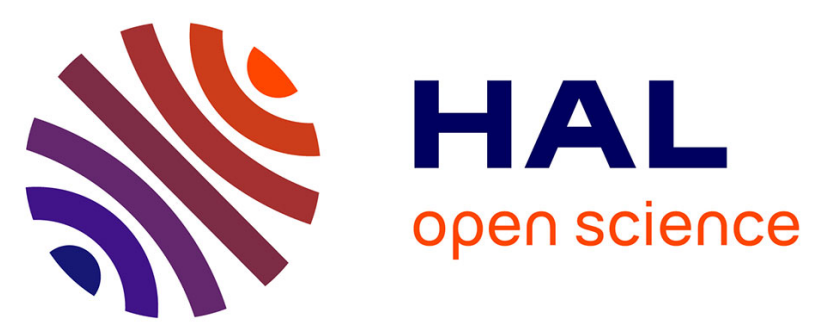

\title{
Circumventing Curse of Dimensionality in the Solution of Highly Multidimensional Models Encountered in Quantum Mechanics Using Meshfree Finite Sums Decomposition
}

Amine Ammar, Francisco Chinesta

\section{To cite this version:}

Amine Ammar, Francisco Chinesta. Circumventing Curse of Dimensionality in the Solution of Highly Multidimensional Models Encountered in Quantum Mechanics Using Meshfree Finite Sums Decomposition. Lecture Notes on Computational Science and Engineering, Springer, pp.1-17, 2008, Meshfree Methods for Partial Differential Equations IV, 10.1007/978-3-540-79994-8_1 . hal-00289677

\section{HAL Id: hal-00289677 \\ https://hal.science/hal-00289677}

Submitted on 13 Feb 2018

HAL is a multi-disciplinary open access archive for the deposit and dissemination of scientific research documents, whether they are published or not. The documents may come from teaching and research institutions in France or abroad, or from public or private research centers.
L'archive ouverte pluridisciplinaire $\mathbf{H A L}$, est destinée au dépôt et à la diffusion de documents scientifiques de niveau recherche, publiés ou non, émanant des établissements d'enseignement et de recherche français ou étrangers, des laboratoires publics ou privés. 


\title{
Circumventing Curse of Dimensionality in the Solution of Highly Multidimensional Models Encountered in Quantum Mechanics Using Meshfree Finite Sums Decomposition
}

\author{
Amine Ammar ${ }^{1}$ and Francisco Chinesta ${ }^{2}$ \\ 1 Laboratoire de Rhéologie, UJF - INPG - CNRS (UMR 5520) \\ 1301 Rue de la Piscine, \\ BP 53 Domaine Universitaire, F-38041 Grenoble cedex 9, France \\ Amine. Ammar@ujf-grenoble.fr \\ 2 Laboratoire de Mécanique des Systèmes et des Procédés, \\ ENSAM - CNRS (UMR 8106) \\ 151 Boulevard de l'Hôpital, F-75013 Paris, France \\ francisco.chinesta@paris.ensam.fr
}

Summary. The fine description of the mechanics and structure of materials at nanometric scale introduces some specific challenges related to the impressive number of degrees of freedom required due to the highly dimensional spaces in which those models are defined. This is the case of quantum mechanics models, in which the wavefunction is defined in a space of dimension $3 \times N_{p}$, being $N_{p}$ the number of particles involved, that leads to the terrific curse of dimensionality. Despite the fact that spectacular progresses have been accomplished in the context of computational mechanics in the last decade, the treatment of those models, as we describe in the present work, needs further developments.

Key words: Quantum Mechanics, Curse of dimensionality, Schrödinger equation, Separated representations

\section{Introduction}

The brut force approach cannot be envisaged for solving highly dimensional models. Some recent approaches allowed computing models defined in moderate multidimensional spaces, as was the case of the sparse grid techniques [4]. However, when the space dimension increases significantly, its treatment becomes delicate. Some specialists as the Nobel Prize R.G. Laughlin, affirmed that no computer existing, or that will ever exist, can break the barriers found in quantum mechanics because it is a catastrophe of dimension [9]. 
We can understand the catastrophe of dimension by assuming a model defined in a hyper-cube in a space of dimension $D, \Omega=]-L, L[D$. Now, if we define a grid to discretize the model, as it is usually performed in the vast majority of numerical methods (finite differences, finite elements, finite volumes, spectral methods etc.), consisting of $N$ nodes on each direction, the total number of nodes will be $N^{D}$. If we assume that for example $N \approx 10$ (an extremely coarse description) and $D \approx 80$ (much lower than the usual dimensions required in quantum or statistical mechanics), the number of nodes involved in the discrete model reaches the astronomical value of $10^{80}$ that represents the presumed number of elementary particles in the universe!. We shall come back to the analysis of these systems later.

Thus, progresses on this field need further developments on the physical modelling as well as the introduction of new ideas and methods in the context of computational physics. In this work we present a recent solution procedure based on the use of a finite sums decomposition, that leads to a separated representation of the involved unknown fields. This solution strategy was successfully applied in [1] [2] for solving different highly dimensional models (involving hundreds of dimensions) encountered in the kinetic theory modelling of complex fluids. In this paper we analyze the suitability of its extension for treating some simple quantum systems. We will prove that the main difficulty related to the solution of the Schrödinger equation for fermions is more in the antisymmetry constraint that the Pauli's principle implies, than in its highly dimensional character.

\subsection{Quantum systems}

The quantum state of a given electronic distribution could be determined by solving the Schrödinger equation. This equation has been for longtime considered as one of the finest descriptions of the world. However, before focusing on the challenges of its numerical solution, we would like to recall that this equation is not relativistic and then it fails when it is applied to describe heavy atoms. Moreover the Pauli's principle constraint was introduced in the Schrödinger formalism in an "ad hoc" way, being the reason of the main numerical difficulties. In our opinion, the best description lies in the solution of the relativistic Dirac's equation (in which the Pauli's principle is implicitly taken into account) within the framework of the quantum field theory. However, the number of works addressing the solution of that equation is nowadays quite reduced.

Some simplificative hypotheses are usually introduced, as for example the Born-Oppenheimer that states that the nuclei can be in first approximation assumed as classical point-like particles, that the state of electrons only depends on the nuclei positions and that the electronic ground state corresponds to the one that minimizes the electronic energy for a nuclear configuration. This equation defines a multidimentionnal problem whose dimension increases linearly with the number of the electrons in the system. 
Thus, the knowledge of a quantum system reduces to the determination of the wavefunction $\Psi\left(\mathbf{x}_{1}, \mathbf{x}_{2}, \cdots, \mathbf{x}_{N}, t ; \mathbf{X}_{1}, \cdots, \mathbf{X}_{M}\right)$ (that establishes that the electronic wavefunction depends parametrically on the nuclei positions) whose evolution is governed by the Schrödinger equation:

$$
i \hbar \frac{\partial \Psi}{\partial t}=-\frac{\hbar^{2}}{2 m_{e}} \sum_{e=1}^{e=N} \nabla_{e}^{2} \Psi+\sum_{e=1}^{e=N-1} \sum_{e^{\prime}=e+1}^{e^{\prime}=N} V_{e e^{\prime}} \Psi+\sum_{e=1}^{e=N} \sum_{n=1}^{n=M} V_{e n} \Psi
$$

where $N$ is the number of electrons and $M$ the number of nuclei, the last ones assumed located and fixed at positions $\mathbf{X}_{j}$. Each electron is defined in the whole physical space $\mathbf{x}_{j} \in \mathbb{R}^{3}, i=\sqrt{-1}, \hbar$ represents the Planck's constant divided by $2 \pi$ and $m_{e}$ is the electron mass.

The differential operator $\nabla_{e}^{2}$ is defined in the conformation space of each particle, i.e.: $\nabla_{e}^{2} \equiv \partial^{2} / \partial x_{e}^{2}+\partial^{2} / \partial y_{e}^{2}+\partial^{2} / \partial z_{e}^{2}$. The Coulomb's potentials accounting for the electron-electron and electron-nuclei interactions writes:

$$
\begin{gathered}
V_{e e^{\prime}}=\frac{\left(q_{e}\right)^{2}}{\left\|\mathbf{x}_{e}-\mathbf{x}_{e^{\prime}}\right\|} \\
V_{e n}=-\frac{q_{n} q_{e}}{\left\|\mathbf{x}_{e}-\mathbf{X}_{n}\right\|}
\end{gathered}
$$

where the electron charge is represented by $q_{e}$ and the nuclei charge by $q_{n}=$ $\left|q_{e}\right| \times Z$ ( $Z$ being the atomic number).

The time independent Schrödinger equation (from which one could determine the ground state, perform quantum static computations or accomplishing separated representations of the time-dependent solution) writes:

$$
-\frac{\hbar^{2}}{2 m_{e}} \sum_{e=1}^{e=N} \nabla_{e}^{2} \Psi+\sum_{e=1}^{e=N-1} \sum_{e^{\prime}=e+1}^{e^{\prime}=N} V_{e e^{\prime}} \Psi+\sum_{e=1}^{e=N} \sum_{n=1}^{n=M} V_{e n} \Psi=E \Psi
$$

where the ground state corresponds to the eigenfunction $\Psi_{0}$ associated with the most negative eigenvalue $E_{0}$.

Several techniques have been proposed for solving this equation. Some of them lie in the direct solution of the (time-independent or time-dependent) Schrödinger equation. Due to the curse of dimensionality its solution is only possible for very reduced populations of electrons.

Other solution strategy is based on the Hartree-Fock (HF) approach and its derived approaches (post-Hartree-Fock methods). The main assumption of this approach lies in the approximation of the joint electronic wavefunction (related to the $N$ electrons) as a product of $N 3 D$-functions (the molecular orbitals) verifying the antisymmetry restriction derived from the Pauli's principle. Thus, the original HF approach consists of writing the joint wavefunction from a single Slater's determinant. The Schödinger equation allows computing the $N$ molecular orbitals after solving the resulting strongly non-linear 
problem. This technique has been extensively used in quantum chemistry to analyze the structure and behavior of molecules involving a moderate number of electrons. Of course, the HF assumption represents sometimes a too crude approximation which invalidate the derived results.

To circumvent this crude approximation different multi-determinant approaches have been proposed. Interested readers can refer the excellent overview of Cancès et al. [5] as well as the different chapters of the handbook on computational chemistry [8]. The simplest possibility consists in writing the solution as a linear combination of some Slater determinants built by combining $n$ molecular orbitals, with $n>N$. These molecular orbitals are assumed known (e.g. the orbitals related to the hydrogen atom) and the weights are searched to minimize the electronic energy. When the molecular orbitals are built from the Hartree-Fock solution (by employing the ground state and some excited eigenfunctions) the technique is known as Configuration Interaction method (CI). A more sophisticated technique consists in writing this many-determinants approximation of the solution by using a number of molecular orbitals $n$ (with $n>N$ ) assumed unknown. Thus, the minimization of the electronic energy leads to compute simultaneously the molecular orbitals as well as the associated coefficients of this many-determinants expansion. Obviously, each one of these unknown molecular orbitals are expressed in an appropriate functional basis (e.g. gaussian functions, ...). This strategy is known as Multi-Configuration Self-Consistent Field (MCSCF).

All the just mentioned strategies (and others like the coupled cluster or the Moller-Plesset perturbation methods) belong to the family of the wavefunction based methods. In any case all these methods can be only used to solve quantum systems composed of a moderate number of electrons. As we confirm later the main difficulty is not in the dimensionality of the space, but in the use of the Slater determinants (needed to take into account the Pauli's principle) whose complexity scales on the factorial of the number of electrons, i.e. in $N$ !.

The second family of approximation methods, widely used in quantum systems composed of hundreds, thousands and even millions of electrons, are based on the density functional theory (DFT). These models, more than looking for the expression of the wavefunction (with the associated multidimensional issue) look for the electronic distribution $\rho(\mathbf{x})$ itself. The main difficulties of this approach are related to the expressions of both the kinetic energy of electrons and the inter-electronic repulsion energy. The second term is usually modelled from the electrostatic self-interaction energy of a charge distribution $\rho(\mathbf{x})$. On the other hand the kinetic energy term is also evaluated in an approximate manner (from the electronic distribution itself in the Thomas-Fermi and related orbital-free DFT models or from a system of $N$ non-interacting electrons -Kohn-Sham models-). Obviously, due to the just referred approximations introduced in the kinetic and inter-electronic interaction energies, a correction term is needed, the so-called exchange-correlationresidual-kinetic energy. However, no exact expression of this correction term 
exists and then different approximate expressions have been proposed and used. Thus, the validity and accuracy of the computed results will depend on the accuracy of the the exchange-correlation term that must be fitted for each system.

The models related to the Thomas-Fermi, less accurate in the practice because the too phenomenological expression of the kinetic energy coming from the reference system of an uniform non-interacting electron gas, allows to consider large multi-electronic systems. In a recent work, Gavini et al. [7] performed multi-million atom simulations by employing the Thomas-FermiWeizsacker family of orbital-free kinetic energy functionals. On the other hand, the Kohn-Sham based models are a priori more accurate, but they need the computation of the $N$ eigenfunctions related to the $N$ lowest eigenvalues of a non-physical atom composed of $N$ non-interacting electrons. In [6] this last approach was considered, and enhanced numerical strategies based on the partition of unity paradigm were introduced.

Transient solutions are very common in the context of quantum gas dynamics (physics of plasma) but are more infrequent in material science when the structure and properties of molecules or crystals are concerned. For this reason, in what follows, we are focusing on the solution of the timeindependent Schrödinger equation which leads to the solution of the associated multidimensional eigenproblem, whose eigenfunction related to the most negative eigenvalue constitutes the ground state of the system.

Quantum chemistry calculations performed in the Born-Oppenheimer setting consist either (i) in solving the geometry optimization problem, that is, to compute the equilibrium molecular configuration (nuclei distribution) that minimizes the energy of the system, finding the most stable molecular configuration that determines numerous properties like for instance infrared spectrum or elastic constants; or (ii) in performing an "ab initio" molecular dynamics simulation, that is, to simulate the time evolution of the molecular structure according to the Newton law of classical mechanics. Molecular dynamics simulations allow to compute various transport properties (thermal conductivity, viscosity, ...) as well as some others non-equilibrium properties.

\subsection{From "ab initio" to molecular dynamics}

Depending on the choice of the method, on the accuracy required, and on the computer facility available, the ab initio methods allow today for the simulations of systems up to ten, one hundred or some million atoms. In time dependent simulations, they are only convenient for small-time simulations, say not more than a picosecond. However, some times larger systems are concerned, and for this purpose one must focus on faster approaches, obviously less accurate. Two possibilities exist: the semi-empirical and the empirical approaches. The semi-empirical approaches speed up the $a b$ initio methods by profiting of the information coming from experiments or previous simulations. 
Empirical methods go on by considering explicitly only the nuclei, by introducing "empirical" potentials leading to the forces acting on the nuclei. Thus, in the stationary setting only the stable configuration is searched, and for this a geometrical optimization (to computed the nuclei equilibrium distribution) is addressed leading to the so-called molecular mechanics. The transient setting results in the classical molecular dynamics but now the computation is speed up of many orders of magnitude with respect to the molecular dynamics where the potentials are computed at the ab initio level.

Thus, if we assume a population of $M$ nuclei (of mass $m_{n}$ ) and a twobody potential (many-body potentials are also available), now the Newton's law writes for a generic nuclei $n$ :

$$
m_{n} \frac{d^{2} \mathbf{X}_{n}}{d t^{2}}=\sum_{k=1, k \neq n} \mathbf{F}_{k}^{n}, \quad \forall n \in[1, \cdots, M]
$$

where $F_{k}^{n}$ denotes the force acting on nucleus $n$ originated by the presence of nucleus $k$. Obviously these forces can be computed from the gradient of the assumed inter-particles potentials.

\section{Solving the time independent Schrödinger equation}

\subsection{Dimensionless form}

The dimensionless form of the Schrödinger equation is performed considering the characteristic time $\frac{\hbar^{3}}{q_{e}^{4} m_{e}}$ and the characteristic length $\frac{\hbar^{2}}{q_{e}^{2} m_{e}}$. The nuclei charge $q_{n}$ becomes dimensionless by using the electron charge $q_{e}$. Thus, for the nuclei, the dimensionless charge results $Z=q_{n} /\left|q_{e}\right|$. For a system consisting of $N$ electrons and $M$ nuclei, the eigenproblem associated with the dimensionless time-independent Schrödinger equation writes:

$$
(\mathcal{H}-E) \Psi=0
$$

or

$$
\left(V\left(\mathbf{x}_{1}, \mathbf{x}_{2}, \cdots, \mathbf{x}_{N_{e}}\right)-E\right) \Psi-\sum_{e=1}^{e=N} \frac{\nabla_{e}^{2} \Psi}{2}=0
$$

where the Hamiltonian writes

$$
\mathcal{H}(\Psi)=-\sum_{e=1}^{e=N} \frac{\nabla_{e}^{2} \Psi}{2}+\underbrace{\sum_{e=1}^{e=N} \sum_{n=1}^{n=M} V_{e n} \Psi+\sum_{e=1}^{e=N-1} \sum_{e^{\prime}=e+1}^{e^{\prime}=N} V_{e e^{\prime}} \Psi}_{V}
$$

where

$$
V_{e e^{\prime}}=\frac{1}{\left\|\mathbf{x}_{e}-\mathbf{x}_{e^{\prime}}\right\|}
$$


and

$$
V_{e n}=-\frac{Z}{\left\|\mathbf{x}_{e}-\mathbf{X}_{n}\right\|}
$$

\subsection{Electronic density and Pauli's exclusion principle}

As soon as the ground state wavefunction is known the electron density associated to the ground state can be computed by applying:

$$
\rho_{e}(\mathbf{x})=\int_{\mathbb{R}^{3 \times(N-1)}}\left|\Psi_{0}\right|^{2} d \mathbf{x}_{1} \cdots d \mathbf{x}_{e-1} d \mathbf{x}_{e+1} \cdots d \mathbf{x}_{N}
$$

that allows computing the electronic density at each point of the space according to:

$$
\rho(\mathbf{x})=\sum_{e=1}^{e=N} \rho_{e}(\mathbf{x})
$$

Probably, the most important difficulty in solving the time-independent Schrödinger equation lies in the fact that the electrons are indistinguishable, that is, they are not labelled. Thus, the many-electrons wavefunction must reflect this fact. If we use $\mathbf{x}_{1}$ and $\mathbf{x}_{2}$ to describe the coordinates of two different electrons, then:

$$
\left|\Psi\left(\mathbf{x}_{1}, \mathbf{x}_{2}, \mathbf{x}_{3}, \cdots, \mathbf{x}_{N}\right)\right|^{2}=\left|\Psi\left(\mathbf{x}_{2}, \mathbf{x}_{1}, \mathbf{x}_{3}, \cdots, \mathbf{x}_{N}\right)\right|^{2}
$$

Thus, if $\Pi$ is any of the $N$ ! permutations of the $N$ electronic coordinates, then:

$$
\Pi\left|\Psi\left(\mathbf{x}_{1}, \mathbf{x}_{2}, \mathbf{x}_{3}, \cdots, \mathbf{x}_{N}\right)\right|^{2}=\left|\Psi\left(\mathbf{x}_{1}, \mathbf{x}_{2}, \mathbf{x}_{3}, \cdots, \mathbf{x}_{N}\right)\right|^{2}
$$

that implies just two possibilities:

$$
\Pi \Psi\left(\mathbf{x}_{1}, \mathbf{x}_{2}, \mathbf{x}_{3}, \cdots, \mathbf{x}_{N}\right)=\Psi\left(\mathbf{x}_{1}, \mathbf{x}_{2}, \mathbf{x}_{3}, \cdots, \mathbf{x}_{N}\right)
$$

or

$$
\Pi \Psi\left(\mathbf{x}_{1}, \mathbf{x}_{2}, \mathbf{x}_{3}, \cdots, \mathbf{x}_{N}\right)=-\Psi\left(\mathbf{x}_{1}, \mathbf{x}_{2}, \mathbf{x}_{3}, \cdots, \mathbf{x}_{N}\right)
$$

The most general statement of the Pauli's exclusion principle for electrons (fermions in the most general case) establishes that an acceptable manyelectrons wavefunction must be antisymmetric with respect to the exchange of the coordinates of any two electrons.

This antisymmetry condition is usually expressed from the Slater's determinant containing all the possible permutations. Thus, if one consider that $F_{1}\left(\mathbf{x}_{1}\right), F_{2}\left(\mathbf{x}_{2}\right), \ldots, F_{N}\left(\mathbf{x}_{N}\right)$ are a set of $N$ functions, each one defined in the space of the associated electron, then an antisymmetric form in the $\mathcal{R}^{3 \times N}$ space is obtained by permuting these functions according to:

$$
\mathcal{A}\left(\prod_{i=1}^{N} F_{i}\left(\mathbf{x}_{i}\right)\right)=\frac{1}{N !}\left|\begin{array}{cccc}
F_{1}\left(\mathbf{x}_{1}\right) & F_{1}\left(\mathbf{x}_{2}\right) & \cdots & F_{1}\left(\mathbf{x}_{N}\right) \\
F_{2}\left(\mathbf{x}_{1}\right) & F_{2}\left(\mathbf{x}_{2}\right) & \cdots & F_{2}\left(\mathbf{x}_{N}\right) \\
\vdots & \vdots & \ddots & \vdots \\
F_{N}\left(\mathbf{x}_{1}\right) & F_{N}\left(\mathbf{x}_{2}\right) & \cdots & F_{N}\left(\mathbf{x}_{N}\right)
\end{array}\right|
$$


In practice, this antisymmetry also affects the spin. For this reason we define the generalized electronic coordinates that involve the physical and the spin coordinates according to:

$$
\left(\mathbf{x}_{i}\right)^{\mathrm{T}}=\left(x_{i}, y_{i}, z_{i}, s_{i}\right)
$$

where $s_{i}$ represents the spin of electron $i$ with two possible values: $s_{i}= \pm s$.

\subsection{Numerical issues related to the treatment of the Coulomb potential}

In order to circumvent the difficulty related to the integration of the inverse of the distance function that appears in the Coulomb inter-electronic potential term in the variational formulation of the Schrödinger equation, two simple alternatives exist: (i) the first one consists in performing an integration of this function in the $6 D$ space in which the integral is defined; and (ii) the second alternative lies in performing a smoothing of the inverse of the distance function using a smoothing parameter $\varepsilon$

$$
\frac{1}{\left\|\mathbf{x}_{e}-\mathbf{x}_{e^{\prime}}\right\|_{\varepsilon}}=\frac{1}{\sqrt{\varepsilon+\left\|\mathbf{x}_{e}-\mathbf{x}_{e^{\prime}}\right\|^{2}}}
$$

We verify numerically that for $\varepsilon<0.01$ the computed solution of the dimensionless Schrödinger equation (that involves a unit characteristic length) were quite similar (with differences lower than one percent). The advantage of this second alternative is that, as described later, that smoothed function can be approximated by a finite sums decomposition (separated representation).

\section{Solution of the multidimensionnal ground state problem}

Firstly, we are describing the numerical procedure without addressing the antisymmetry constraint, even if the spin is explicitly considered. Thus, $\Omega=$ $\mathcal{R}^{3} \times\{-s, s\}$, the whole multidimensional domain being represented by $\Omega^{N}$ $\left(\Omega^{N}=\left(\mathcal{R}^{3} \times\{-s, s\}\right)^{N}\right)$.

We introduce the following notation: $N$ is the number of electrons; $M$ is the number of nuclei; $Q$ is the number of finite sums present in the decomposition of the inverse of the distance function; $n$ is the number of finite sums approximating the wavefunction and $m$ the number of terms representing the different Coulomb potentials $m=N \times(N-1) \times Q+M \times N$.

Now, the problem to be solved writes:

$$
\left(V\left(\mathbf{x}_{1}, \ldots, \mathbf{x}_{N}\right)-E\right) \Psi-\frac{1}{2} \frac{\partial^{2} \Psi}{\partial \partial \mathbf{x}^{2}}=0
$$


with

$$
\int_{\Omega^{N}} \Psi^{2}(\mathbf{x}) d \Omega^{N}=1
$$

where $V$ is the inter-electronic and electron-nucleus potential. These potentials contain two contributions:

$$
\frac{1}{\left\|\mathbf{x}_{i}-\mathbf{x}_{j}\right\|_{\varepsilon}}=\sum_{l=1}^{Q} B_{l}^{1}\left(\mathbf{x}_{i}\right) B_{l}^{2}\left(\mathbf{x}_{j}\right) \prod_{k=1, k \neq i, j}^{N} 1\left(\mathbf{x}_{k}\right)
$$

and

$$
\frac{-Z_{j}}{\left\|\mathbf{x}_{i}-\mathbf{X}_{j}\right\|_{\varepsilon}}=\frac{-Z_{j}}{\left\|\mathbf{x}_{i}-\mathbf{X}_{j}\right\|_{\varepsilon}} \prod_{k=1, k \neq i}^{N} 1\left(\mathbf{x}_{k}\right)
$$

where the nuclei positions $\mathbf{X}_{j}(j=1, \cdots, M)$, are assumed fixed and known, and $1(\mathbf{x})$ is the unit function. Thus, the potential can be written in the general form:

$$
V\left(\mathbf{x}_{1}, \ldots, \mathbf{x}_{N}\right)=\sum_{h=1}^{m} \prod_{k=1}^{N} A_{k}^{h}\left(\mathbf{x}_{k}\right)
$$

The variational formulation of the problem writes:

$$
\int_{\Omega^{N}} \Psi^{*}\left(V\left(\mathbf{x}_{1}, \ldots, \mathbf{x}_{N}\right)-E\right) \Psi+\frac{1}{2} \frac{\partial \Psi^{*}}{\partial \mathbf{x}} \frac{\partial \Psi}{\partial \mathbf{x}} d \Omega^{N}=0
$$

that can be rewritten in the compact form:

$$
\int_{\Omega^{N}} \Psi^{*}(\mathcal{H}-E) \Psi d \Omega^{N}=0
$$

where we assumed that on the boundary of $\Omega^{N}$ the wavefunction normal derivative vanishes.

For solving this problem we firstly apply a fixed point strategy, assuming $E$ known. The solution after $n$ iterations is assumed in the form:

$$
\Psi\left(\mathbf{x}_{1}, \mathbf{x}_{2}, \cdots, \mathbf{x}_{N}\right)=\sum_{j=1}^{n} \alpha_{j} \prod_{k=1}^{N} F_{k j}\left(\mathbf{x}_{k}\right)
$$

Now, we proceed in two steps: a projection and an enrichment stages, both performed in the finite elements framework.

\section{Projection stage:}

Assuming known the functions $F_{k j}\left(\mathbf{x}_{k}\right) \forall j=1, . ., n, \forall k=1, . ., N$, we look for the best coefficients $\alpha_{j}$ of the approximation $\Psi\left(\mathbf{x}_{1}, \cdots, \mathbf{x}_{N}\right)=$ $\sum_{j=1}^{n} \alpha_{j} \prod_{k=1}^{N} F_{k j}\left(\mathbf{x}_{k}\right)$ by enforcing the variational formulation. 


\section{Enrichment stage:}

Now, with the just computed coefficients we look for a new term of the finite sums decomposition, $\prod_{k=1}^{N} R_{k}\left(\mathbf{x}_{k}\right)$, of the wavefunction $\Psi\left(\mathbf{x}_{1}, \cdots\right.$, $\left.\mathbf{x}_{N}\right)$, i.e. $\Psi\left(\mathbf{x}_{1}, \cdots, \mathbf{x}_{N}\right)=\sum_{j=1}^{n} \alpha_{j} \prod_{k=1}^{N} F_{k j}\left(\mathbf{x}_{k}\right)+\prod_{k=1}^{N} R_{k}\left(\mathbf{x}_{k}\right)$, by enforcing again the variational formulation.

To approximate each one of the functions defined in the domain $\Omega$ in which each electron is defined, we introduce the vector $\mathbf{N}$ containing the finite element shape functions defined in $\Omega$. The shape functions derivatives are grouped in $\mathbf{d N}$. The vectors containing the nodal values of functions $F$ and $R$ will be noted by $\mathbf{F}$ and $\mathbf{R}$ respectively.

Before to detail both algorithm steps, we are introducing the matrix form of the integrals involved in the variational formulation:

$$
\mathbb{N}=\int_{\Omega} \mathbf{N} \mathbf{N}^{T} d \Omega, \quad \mathbb{D}=\int_{\Omega} \mathbf{d} \mathbf{N} \mathbf{d} \mathbf{N}^{T} d \Omega, \quad \mathbb{A}_{k}^{h}=\int_{\Omega} A_{k}^{h}(x) \mathbf{N} \mathbf{N}^{T} d \Omega
$$

\subsection{Projection stage}

At this step, the unknowns are the approximation basis function coefficients. The unknown field (the wavefunction) at the present iteration is approximated as:

$$
\Psi\left(\mathbf{x}_{1}, \cdots, \mathbf{x}_{N}\right)=\left[\prod_{k=1}^{N} \mathbf{N}^{T} \mathbf{F}_{k 1}, \cdots, \prod_{k=1}^{N} \mathbf{N}^{T} \mathbf{F}_{k n}\right]\left[\begin{array}{c}
\alpha_{1} \\
\vdots \\
\alpha_{n}
\end{array}\right]
$$

and the associated test field as:

$$
\Psi^{*}\left(\mathbf{x}_{1}, \cdots, \mathbf{x}_{N}\right)=\left[\alpha_{1}^{*}, \cdots, \alpha_{n}^{*}\right]\left[\begin{array}{c}
\prod_{k=1}^{N} \mathbf{F}_{k 1}^{T} \mathbf{N} \\
\vdots \\
\prod_{k=1}^{N} \mathbf{F}_{k n}^{T} \mathbf{N}
\end{array}\right]
$$

Thus, the variational formulation of the eigenproblem writes:

$$
\alpha^{* T}\left(\mathbb{K}_{H}-E \mathbb{K}_{L}\right) \alpha=0
$$

where

$$
\begin{gathered}
\left(\mathbb{K}_{H}\right)_{i, j}=\sum_{h=1}^{m} \prod_{k=1}^{N} \mathbf{F}_{k i}^{T} \mathbb{A}_{k}^{h} \mathbf{F}_{k j}+\frac{1}{2} \sum_{k=1}^{N}\left(\mathbf{F}_{k i}^{T} \mathbb{D} \mathbf{F}_{k j} \prod_{l=1, l \neq k}^{N} \mathbf{F}_{l i}^{T} \mathbb{N F}_{l j}\right) \\
\left(\mathbb{K}_{L}\right)_{i, j}=\prod_{k=1}^{N} \mathbf{F}_{k i}^{T} \mathbb{N F}_{k j}
\end{gathered}
$$


whose solution must be searched under the normality constraint:

$$
\alpha^{T} \mathbb{K}_{L} \alpha=1
$$

that is enforced within an iteration fixed point strategy. Then, a correction of the eigenvalue is performed according to:

$$
E=\frac{\int_{\Omega^{N}} \Psi \mathcal{H} \Psi d \Omega^{N}}{\int_{\Omega^{N}} \Psi \Psi d \Omega^{N}}=\frac{\alpha^{T} \mathbb{K}_{H} \alpha}{\alpha^{T} \mathbb{K}_{L} \alpha}=\alpha^{T} \mathbb{K}_{H} \alpha
$$

\subsection{Basis enrichment stage}

Now, a new optimal product $\prod_{k=1}^{N} R_{k}\left(\mathbf{x}_{k}\right)$ of functions is searched by enforcing the variational formulation:

$$
\Psi\left(\mathbf{x}_{1}, \cdots, \mathbf{x}_{N}\right)=\sum_{j=1}^{n} \alpha_{j} \prod_{k=1}^{N} F_{k j}\left(\mathbf{x}_{k}\right)+\prod_{k=1}^{N} R_{k}\left(\mathbf{x}_{k}\right)
$$

The fixed point strategy is used again for computing each function $R_{d}\left(\mathbf{x}_{d}\right)$ involved in the product, by assuming known the remaining $N-1$ functions. Thus, the test functions are expressed as:

$$
\Psi^{*}\left(\mathbf{x}_{1}, \cdots, \mathbf{x}_{N}\right)=R_{d}^{*}\left(\mathbf{x}_{d}\right) \prod_{k=1, k \neq d}^{N} R_{k}\left(\mathbf{x}_{k}\right)
$$

that introduced in the vartiational formulation leads to the linear system:

$$
\mathbf{R}_{d}^{*} \mathbf{V}\left(\mathbf{R}_{1}, \cdots, \mathbf{R}_{N}\right)+\mathbf{R}_{d}^{*} \mathbb{K}\left(\mathbf{R}_{1}, \cdots, \mathbf{R}_{N}\right) \mathbf{R}_{d}=\mathbf{0}
$$

where

$$
\begin{aligned}
\mathbb{K}=(-E \mathbb{N} & \left.+\frac{1}{2} \mathbb{D}\right) \prod_{k=1, k \neq d}^{N} \mathbf{R}_{k}^{T} \mathbb{N R}_{k}+\sum_{h=1}^{m} \mathbb{A}_{d}^{h} \prod_{k=1, k \neq d}^{N} \mathbf{R}_{k}^{T} \mathbb{A}_{k}^{h} \mathbf{R}_{k}+ \\
& +\frac{1}{2} \sum_{l=1, l \neq d}^{N} \mathbb{N}\left(\mathbf{R}_{l}^{T} \mathbb{D} \mathbf{R}_{l}\right) \prod_{k=1, k \neq d, l}^{N} \mathbf{R}_{k}^{T} \mathbb{N}_{k}
\end{aligned}
$$

and

$$
\begin{aligned}
\mathbf{V}=\sum_{j=1}^{n} \alpha_{j} & \left(\left(-E \mathbb{N}_{d j}+\frac{1}{2} \mathbb{D} \mathbf{F}_{d j}\right) \prod_{k=1, k \neq d}^{N} \mathbf{R}_{k}^{T} \mathbb{N}_{k j}+\right. \\
& +\sum_{h=1}^{m} \mathbb{A}_{d}^{h} \mathbf{F}_{d j} \prod_{k=1, k \neq d}^{N} \mathbf{R}_{k}^{T} \mathbb{A}_{k}^{h} \mathbf{F}_{k j}+
\end{aligned}
$$




$$
\left.+\frac{1}{2} \sum_{l=1, l \neq d}^{N} \mathbb{N F}_{d j}\left(\mathbf{R}_{l}^{T} \mathbb{D}_{l j}\right) \prod_{k=1, k \neq d, l}^{N} \mathbf{R}_{k}^{T} \mathbb{N F}_{k j}\right)
$$

solved again under the non-linear normality constraint:

$$
\mathbf{R}_{d}^{T} \mathbb{N R}_{d}=1
$$

that is enforced within an iteration fixed point scheme that update at each iteration the eigenvalue.

\subsection{Solution algorithm}

The solution algorithm can be summarized as follows:

- Let $E=E_{0}$ be the first trial eigenvalue.

- Compute until convergence the following steps:

1. Proceed with the enrichment step to compute $\mathbf{R}_{k}, \forall k$. Note that at the first iteration $\mathbf{V}=0$.

2. Update the approximation basis with the just computed functions.

3. Proceed with the projection step.

4. Updated the eigenvalue $E$.

\subsection{Computing the electronic density}

For each electron $k$ we can compute its spatial distribution from:

$$
\rho_{k}(\mathbf{x})=\sum_{i=1}^{n} \sum_{j=1}^{n} \alpha_{i} \alpha_{j} F_{k i}(\mathbf{x}) F_{k j}(\mathbf{x}) \prod_{l=1, l \neq k}^{N} \mathbf{F}_{l i}^{T} \mathbb{N} \mathbf{F}_{l j}
$$

The total electronic density is then obtained by adding the spatial distribution related to each electron.

\subsection{Taking into account the antisymmetry}

In order to compute an antisymmetric solution we could apply the just proposed algorithm applying an antisymmmetrizer after each enrichment step. This results equivalent to enrich with the $N$ ! functions derived from the product of functions just computed in the enrichment step, via the Slater's determinant. This strategy converges slowly and requires the storage of numerous functions.

An alternative procedure proposed by Beylkin and Mohlenkamp [3] allows alleviating the storage cost. Since the operator $(\mathcal{H}-E)$ is purely symmetric it could commute with the antisymmetrizer $\mathcal{A}$. Thus, we can defer the application of the antisymmetrizer and the operator. The idea is to incorporate the 
antisymmetrizer in the algorithm by evaluating its effect. Of coarse the pseudowave functions obtained are not the solution of the schrodinger equation and need to be antisymmetrized to obtain the exact solution.

In this work the antisymmetrizer operator will apply on the test functions. Thus, the variational formulation writes:

$$
\int_{\Omega^{N}}\left(\mathcal{A} \Psi^{*}\right)(\mathcal{H}-E) \Psi d \Omega^{N}=0
$$

that requires the modification of the test function approximation at the projection step:

$$
\mathcal{A} \Psi^{*}\left(\mathbf{x}_{1}, \cdots, \mathbf{x}_{N}\right)=\left[\alpha_{1}^{*}, \cdots, \alpha_{n}^{*}\right]\left[\begin{array}{c}
\mathcal{A} \prod_{k=1}^{N} \mathbf{F}_{k 1}^{T} \mathbf{N} \\
\vdots \\
\mathcal{A} \prod_{k=1}^{N} \mathbf{F}_{k n}^{T} \mathbf{N}
\end{array}\right]
$$

as well as at the enrichment step:

$$
\mathcal{A} \Psi^{*}\left(\mathbf{x}_{1}, \cdots, \mathbf{x}_{N}\right)=\mathcal{A}\left[R_{d}^{*}\left(x_{d}\right) \prod_{k=1, k \neq d}^{N} R_{k}\left(\mathbf{x}_{k}\right)\right]
$$

Thus, the algorithm remains basically unchanged, but an additional condition at the enrichment step must be introduced. In fact, if the $N$ functions $F_{k j}$ $(j=1, . ., n)$ are linearly dependent, then the determinant operator vanishes. Thus, an orthogonalization is performed at each enrichment step according to:

$$
\begin{gathered}
<R_{d}\left(\mathbf{x}_{d}\right), R_{k}\left(\mathbf{x}_{k}\right)>=R_{d}^{T}\left(\mathbf{x}_{d}\right) \mathbb{N} R_{k}\left(\mathbf{x}_{k}\right)=0 \\
\forall d=1, \cdots, N ; \quad \forall k=1, \cdots, d-1, d+1, \cdots, N
\end{gathered}
$$

\section{Some preliminary numerical results}

To illustrate the solution procedure we start solving systems composed of a single nucleus $(Z=3$ ) and different number of electrons (from 1 to 5 ). Figure 4.1 depicts, assuming a one-dimensional physical space, the electronic distributions as well as the differences between each couple of consecutive electronic distributions. This simulation was carried out by assuming a large enough one-dimensional domain such that both the electronic distribution and its derivative vanish on its boundary. Obviously, the numerical model could be improved by using larger domains and non-uniform one-dimensional nodal distributions, but in this first attempt we considered the simplest strategy. The length of the computational domain was set to 10 dimensionless units as depicted in figure 4.1.

We can notice that the first two electrons are occupying a $s$-type orbital. When an additional electron is introduced, and due to the Pauli's exclusion 

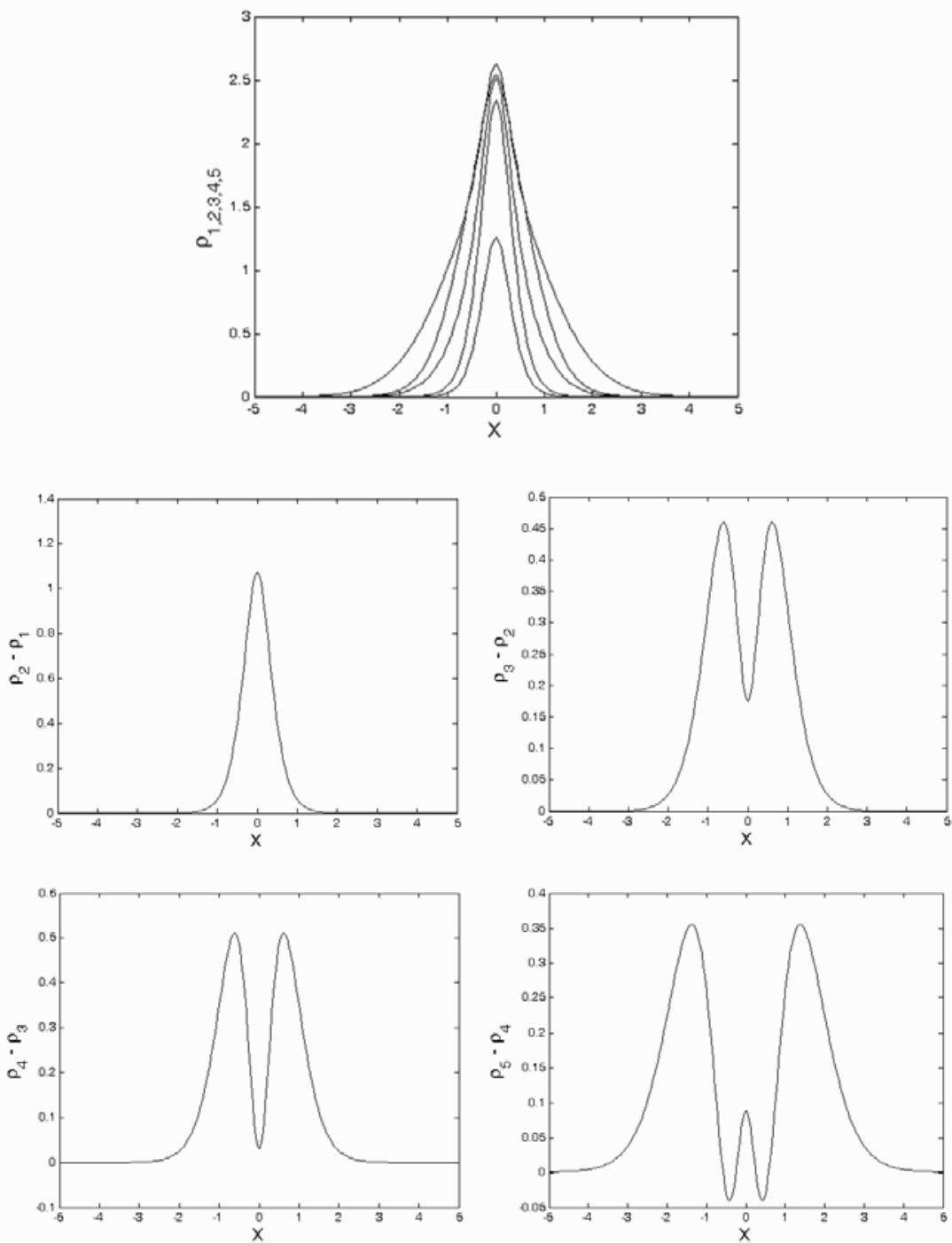

Figure 4.1. Evolution of the electronic distribution of a system composed of a single nucleus $(Z=3)$ and an increasing number of electrons (one-dimensional physical space).

principle, the three electrons cannot occupy the same orbital. Thus, a kind of $p$-orbital is encountered. This behavior is also noticed when $2 D$ physical spaces are considered, as figure 4.2 illustrates.

Now, we are considering the hypothetical one-dimensional molecules of helium $\left(\mathrm{He}_{2}\right)$ and of $\mathrm{LiH}$. For this purpose we consider the system composed 

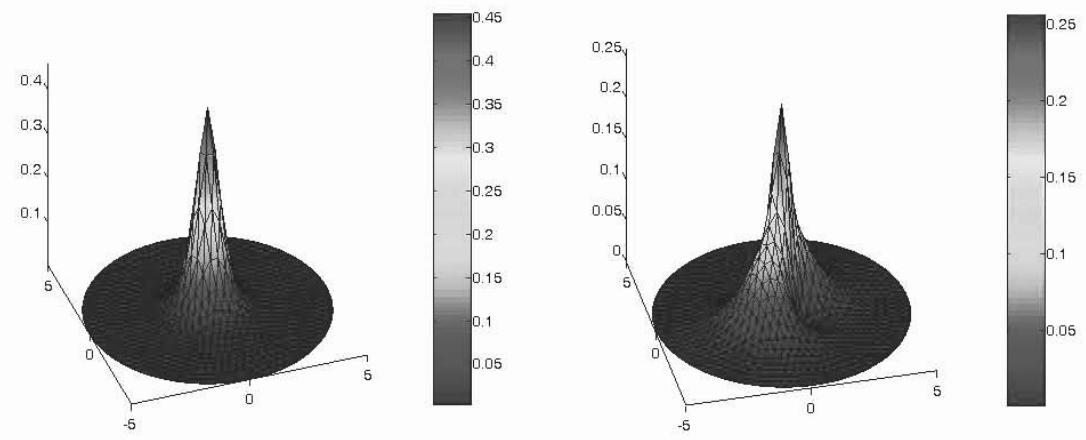

Figure 4.2. Evolution of the electronic distribution of a system composed of a single nucleus $(Z=3)$ and an increasing number of electrons (two-dimensional physical space). (See also Color Plate on page 359)

of 4 electrons and two nuclei. Now, the total energy (which is the sum of the ground state energy plus the one associated to the inter-nuclei Coulomb's potential) is obtained as a function of the distance between both nuclei. The computational domain consists of 16 dimensionless units (that seems to be large enough to ensure the nullity of both the electronic distribution and its derivative on the domain boundary for all the relative positions between the nuclei later considered).

The computed evolution is depicted in 4.3(a) that explain the higher stability of the $\mathrm{LiH}$ molecule. In the same figure we depict the electronic distribution when the inter-nuclei distance takes the dimensionless values of 0.5 , $1,2,3$ and 4 . When the distance increases we can notice that the electronic distribution for the $\mathrm{He}_{2}$ remains symmetric. On the contrary, an asymmetric charge distribution is noticed in the case of the $\mathrm{LiH}$ molecule.

In all the simulations reported in this section we considered the usual 3D Coulomb potential that was simply and crudely restricted to $1 \mathrm{D}$ or $2 \mathrm{D}$, even if this reduction has not any physical meaning. The smoothing parameter $\epsilon$ in Eq. (2.19) was set to $\epsilon=0.01$. Different separated representations of the inverse of the distance function (according to Eq. (3.22)) were performed by increasing the approximation accuracy. Thus, the number of finite sums used in the approximation (3.22) ranged in the interval $Q \in[100,300]$. Finally, in all the simulations that were performed the accurate representation of wavefunctions needed for the use of around ten finite sums, i.e. in Eq. (3.27) $n$ was $n \approx 10$. 

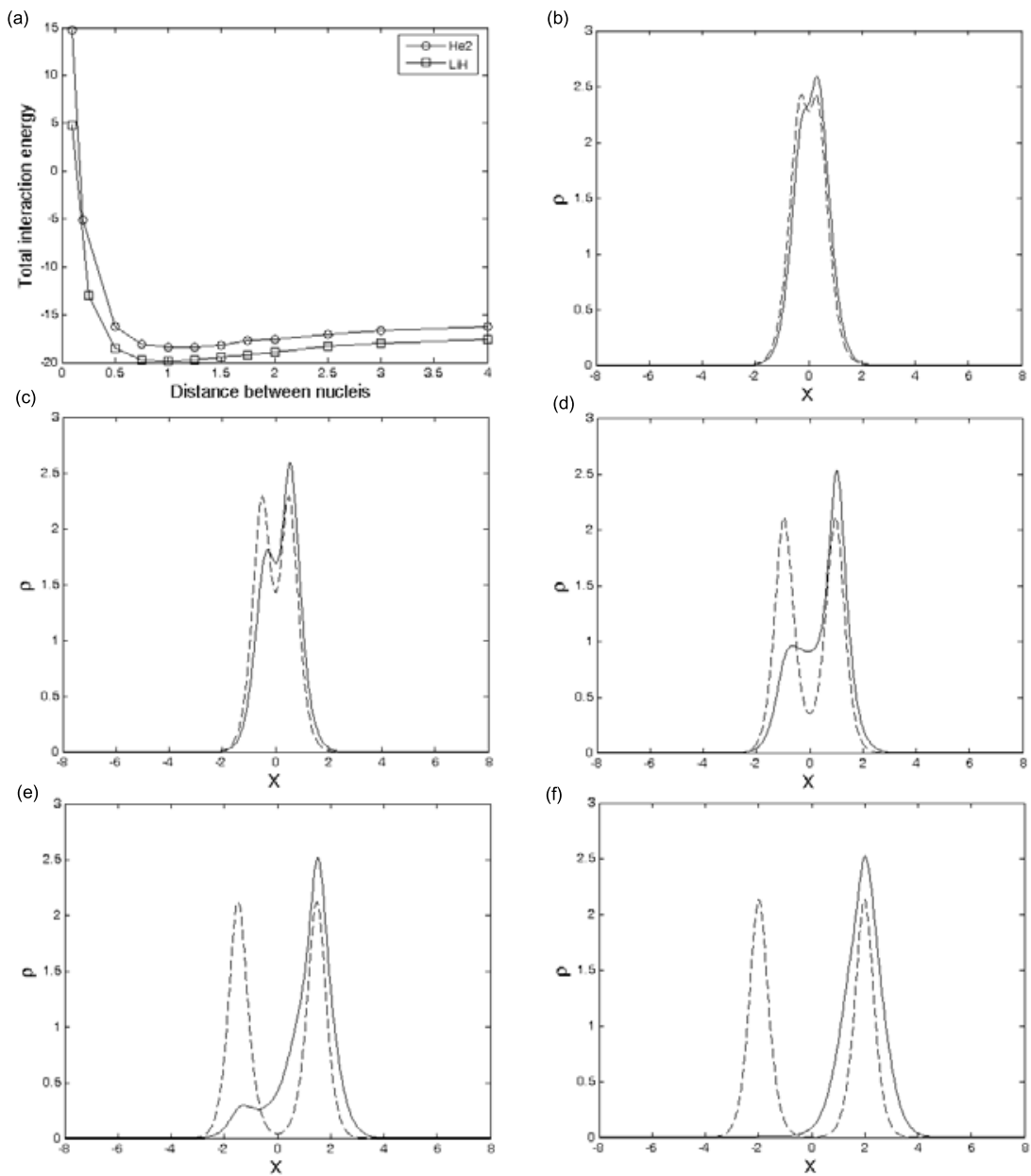

Figure 4.3. Analysis of the hypothetical one-dimensional $\mathrm{LiH}$ and $\mathrm{He}_{2}$ molecules.

\section{Conclusions}

This paper analyzed the suitability of a finite sums decomposition based on a separated representation, to address highly dimensional models, as the ones encountered in quantum mechanics when the solution of the Schrödinger equation is envisaged.

Based in our former experience on the solution of models defined in highly dimensional spaces (involving hundreds of dimensions) we have extended the numerical technique that we proposed in [1] [2] to the direct solution of the Schrödinger equation. 
The main conclusion of this analysis was that the main difficulty related to the solution of the Schrödinger equation for fermions is more the antisymmetry constraint that the Pauli's principle implies, than its highly dimensional character. The curse of dimensionality can be circumvented efficiently using finite sums decompositions based on separated representations as we proved in [1] [2]. However an efficient treatment of the antisymmetry constraint needs for further developments, if one want to address the direct solution of the Schrödinger equation. One possibility is to focus on the improvement of approximated approaches (the ones derived from the DFT or the Hartree-Fock approaches). Others alternatives need further developments.

\section{References}

1. A. Ammar, B. Mokdad, F. Chinesta, R. Keunings, A new family of solvers for some classes of multidimensional partial differential equations encountered in kinetic theory modeling of complex fluids, Journal of Non-Newtonian Fluid Mechanics, 139, 2006, 153-176.

2. A. Ammar, B. Mokdad, F. Chinesta, R. Keunings, A new family of solvers for some classes of multidimensional partial differential equations encountered in kinetic theory modeling of complex fluids. Part II: transient simulation using space-time separated representation, Journal of Non-Newtonian Fluid Mechanics, 144, 2007, 98-121.

3. G. Beylkin, M.J. Mohlenkamp, Algorithm for numerical analysis in high dimensions, SIAM J. Sci. Comput., 26/6, 2005, 2133-2159.

4. H.J. Bungartz, M. Griebel, Sparse grids, Acta Numer., 13, 2004, pp. 1-123.

5. E. Cancès, M. Defranceschi, W. Kutzelnigg, C. Le Bris, Y. Maday, Computational Quantum Chemistry: a primer, Handbook of Numerical Analysis, Vol. X, Elsevier, 2003, pp. 3-270.

6. J.S. Chen, W. Hu, M. Puso, Orbital HP-clouds for solving Schrdinger equation in quantum mechanics, Comput. Methods Appl. Mech. Engrg., 196/37-40, 2007, pp. 3693-3705.

7. V. Gavini, K. Bhattacharya, M. Ortiz, Quasi-continuum orbital-free densityfunctional theory: A route to multi-million atom non-periodic DFT calculation, Journal of the Mechanics and Physics of Solids, 55/4, 2007, pp. 697-718.

8. Handbook of Numerical Analysis, Vol. X: Computational Chemistry, C. Le Bris editor, Elsevier, 2003.

9. R.B. Laughlin, The theory of everything, Proceeding of the U.S.A. National Academy of Sciences, 2000. 